U.S and explore sociodemographic, healthcare, and diseaserelated factors that can impact the risk of depression in this cohort.

Methods We conducted a cross-sectional study in a predominantly African American cohort of patients with primary CCLE from the Southeast U.S. Participants were assessed about depression, skin-related quality of life, sociodemographic and healthcare factors using validated self-reported tools. Depression was assessed using the PROMIS depression short form 8a. We conducted Student-T test and ANOVA to examine the severity of depression symptoms across sociodemographic subgroups, and we further explored factors associated with depression using univariate and multivariate analyses.

Results Among 106 patients with a documented diagnosis of primary CCLE, 28 (26.4\%) had moderate to severe depression. The univariate analysis showed that being employed and insured, reporting higher social support, visiting a primary care physician in the last year, and reporting better physicianpatient communication protected against depression. Patient's perceptions of staff disrespect and greater burden of the skin condition on patient's quality of life corresponded with higher risk. In the multivariate analysis, staff disrespect and emotional support were the only factors that increased and reduced the odds of depression, respectively (Staff disrespect: OR 2.35 [95\% CI 1.06 to 5.17] per 1-unit score increase; emotional support: OR $0.480 .35-0.66]$ per 5 -unit score increase).

Conclusions Patients with isolated CCLE experience higher rates of depression than the general population. This population may uniquely benefit from routine mental health care such as depression screening and management. Moreover, office staff can undergo educational sessions to ensure fair treatment of all patients, and clinical tools may be implemented to assess patients' emotional support and initiate referral to care-coordinators, support groups, primary care and psychotherapy or psychiatric care as needed.

Acknowledgements This study is supported by the Centers for Disease Control and Prevention (CDC) Grant 1U01DP005119.

The findings and conclusions in this report are those of the author(s) and do not necessarily represent the official position of the CDC.

\section{LL-03 PATIENT-REPORTED OUTCOMES AMONG PEDIATRIC LUPUS NEPHRITIS PATIENTS TREATED WITH CARRA LUPUS NEPHRITIS CONSENSUS TREATMENT PLANS}

${ }^{1}$ Jennifer C Cooper, ${ }^{2}$ Kelly Rouster-Stevens, ${ }^{3}$ Tracy Wright, ${ }^{4}$ Joyce Hsu, ${ }^{5}$ Marisa S KleinGitelman, ${ }^{6}$ Stacy P Ardoin, ${ }^{7}$ Laura E Schanberg, ${ }^{8}$ Hermine Brunner, ${ }^{9} \mathrm{~B}$ Anne Eberhard, ${ }^{10}$ Linda Wagner-Weiner, ${ }^{1}$ Emily von Scheven*, for the CARRA Registry Investigators. ${ }^{1}$ University of California, San Francisco, USA; ${ }^{2}$ Emory University School of Medicine, USA; ${ }^{3}$ Texas Scottish Rite Children's Hospital, USA; ${ }^{4}$ Stanford University, USA; ${ }^{5}$ Ann and Robert H. Lurie Children's Hospital of Chicago, USA; ${ }^{6}$ Ohio State University College of Medicine, USA; ${ }^{7}$ Duke University Medical Center, USA; ${ }^{8}$ Cincinnati Children's Hospital Medical Center, USA; ${ }^{9}$ Cohen Children's Hospital Medical Center, USA; ${ }^{10}$ University of Chicago Hospitals, USA

\subsection{6/lupus-2018-Ism.113}

Background The Childhood Arthritis and Rheumatology Research Alliance (CARRA) developed consensus treatment plans (CTP) for childhood proliferative lupus nephritis (LN) induction therapy to reduce treatment variability and support comparative effectiveness research comparing efficacy and tolerability of Mycophenolate mofetil (MMF) and intravenous cyclophosphamide (IV CYC). The CTPs specify two immunosuppression regimens (IV CYC, oral MMF) and three corticosteroid regimens (primarily oral, primarily IV, and mixed oral/IV). In addition to provider-reported outcomes, we assessed patient-reported outcomes in a pilot observational study.

Methods We enrolled 41 subjects with childhood SLE from 10 CARRA sites. Subjects had new-onset biopsy proven proliferative LN and were starting MMF or IV CYC. We collected baseline demographics, disease-related features and patientreported outcomes, including functional disability (Childhood Health Assessment Questionnaire (CHAQ), range 0-3), Health-related quality of life (HRQOL) (Child Health Questionnaire (CHQ), excellent, very good, good, poor, very poor), well-being (CHQ, range 0-10), and pain (CHAQ, 010). With the exception of pain, parents reported outcomes for subjects $<10$ years of age. We report baseline and 12 month follow-up results.

Results The majority of participants were female (83\%), mean age was 14 years (SD 2.6). At baseline, SLEDAI ranged from 2-34 (median 13, IQR 10-21) and glomerular filtration rate ranged from 41-151 ml/min/1.73 $\mathrm{m}^{2}$ (median 94, IQR 70107). Baseline functional ability (CHAQ) ranged from $0-1.75$ $(\mathrm{n}=38)$ and was abnormal (CHAQ $>0)$ in $55.3 \%$ of subjects. By 12 months, there was improvement with 14.8\% reporting abnormal functional ability. HRQOL at baseline ranged from excellent to poor (13\% excellent, $37 \%$ very good, $45 \%$ good, $5 \%$ poor, $\mathrm{n}=38)$ and was improved at 12 months $(32 \%$ excellent, $48 \%$ very good, $16 \%$, good, $4 \%$ poor, $n=25$, see figure 1). Baseline median parent-reported overall wellbeing was 3.0 (IQR 2-5), with 21\% reporting doing 'very well' (score=0). At 12 months, overall wellbeing had improved (median 0.5, IQR 0-3) with 50\% reporting doing 'very well'. Baseline patient-reported pain ranged from 0 (no pain) to 10 (very severe pain) (median 1, IQR $0-5, \mathrm{n}=39$ ). Pain was improved at 12 months (range $0-7$, median 0, IQR $0-3, \mathrm{n}=27$ ).

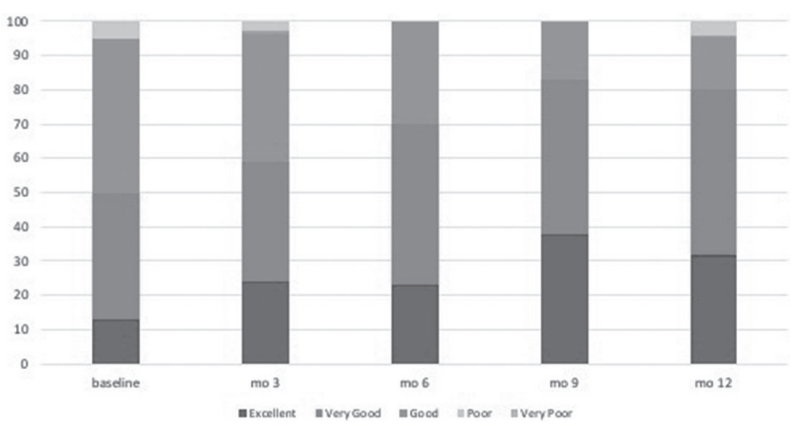

Abstract LL-03 Figure 1 HRQOL over 12 months of follow-up

Conclusions Although diagnosed with a severe medical condition and experiencing functional disability, most children reported good to excellent QOL and only minimal pain. After 12 months, the proportion of patients reporting excellent QOL increased, however reduced QOL persisted for many. Further study is needed to elucidate the factors impacting overall QOL in children with SLE, and association with different treatment strategies.

Acknowledgements We would like to acknowledge the CARRA Registry Investigators, Dr. Marilyn Punaro for her leadership in the CARRA lupus nephritis CTP development process and Thomas Phillips for his data management assistance. This study was supported by funding from the Arthritis Foundation, Lupus Foundation of America, CARRA and NIAMS. 